\title{
Comparison and analysis of Microblog and WeChat based on information spreading models
}

\author{
Juanjuan $\mathrm{He}^{1, \text { a }}$, Tao Wang ${ }^{2, \mathrm{~b}}$ \\ ${ }^{1,2}$ School of Mathematics and Physics, North China Electric Power University, Baoding 071000, \\ China; \\ a1525062812@qq.com, bwtwxx@126.com
}

Keywords: information spreading model, WeChat, Microblog, super spreaders, centralization

\begin{abstract}
The important difference of Microblog network and WeChat network is network centralization. Microblog is a platform which pay attention to very important users, but WeChat is a decentralized network. Based on this point, we firstly build a model called IRC2S information spreading model to simulate information spreading process in WeChat, and it includes five groups: ignorants, reviewers, collectors, sharers and stiflers. Secondly, a group called super spreaders is added to the IRC2S information spreading model, which embody the feature of centralization of Microblog network. In order to approach real information spreading rules of Microblog network, we distinguish the average degrees of ordinary nodes and center nodes which reflect the feature that very important users have a great many of fans. Finally, we perform numerical simulation to the above three models and discover that centralization of network make information's propagation speed faster and information's propagation range wider.
\end{abstract}

\section{Introduction}

In recent years, Microblog and WeChat are very popular online social platforms. However, many focusing events are popped up on Microblog but not on WeChat. We build information spreading models to find the reasons. Many information spreading models are built based on classical epidemic propagation models. Zanette [1] studied epidemic-like rumor spreading process on small-word networks. Zhao et al. [2] added forgetting and remembering mechanisms to rumor spreading model on social networks. In addition, trust mechanism [3] were introduced in the classical epidemic propagation models, which was also applied to complex social networks. As the development of WeChat and Microblog, scholars began to study information spreading model on online social networks. Some scholars built information propagation model in online social networks from a tie-strength perspective [4]. Dynamics of information propagation model with cooperative and competitive mechanisms was applied to online social networks [5]. Zhao et al. [6] put the rumor spreading model with forgetting mechanism applying to new media, e.g. microblogging. Above-mentioned models have universality, but they do not aimed at certain online social network and also can not reflect the difference of different online social platforms. So we will build new models to embody the difference of WeChat and Microblog.

This paper is divided two big parts: description of models and analysis of numerical simulation. In one part, we build three models: IRC $2 S$ information spreading model which is based on WeChat Moments, IRC $3 S$ information spreading model which add a group super spreaders on the basis of IRC2S model, the last one is advanced IRC 3S information spreading model which reflect the effect of super spreaders' degree. The advanced IRC $3 S$ information spreading model is built to simulate information propagating process on Microblog. In other part, we perform numerical simulation to built three models. 


\section{Description of models}

\section{The IRC2S information spreading model}

We build an information spreading model based on WeChat Moments firstly. The population is divided five groups: ignorants (I), reviewers $(\mathrm{R})$, collectors $(\mathrm{C})$, sharers $\left(S_{1}\right)$ and stiflers $\left(S_{2}\right)$. WeChat Moments information spreading model is named IRC2S information spreading model in short.

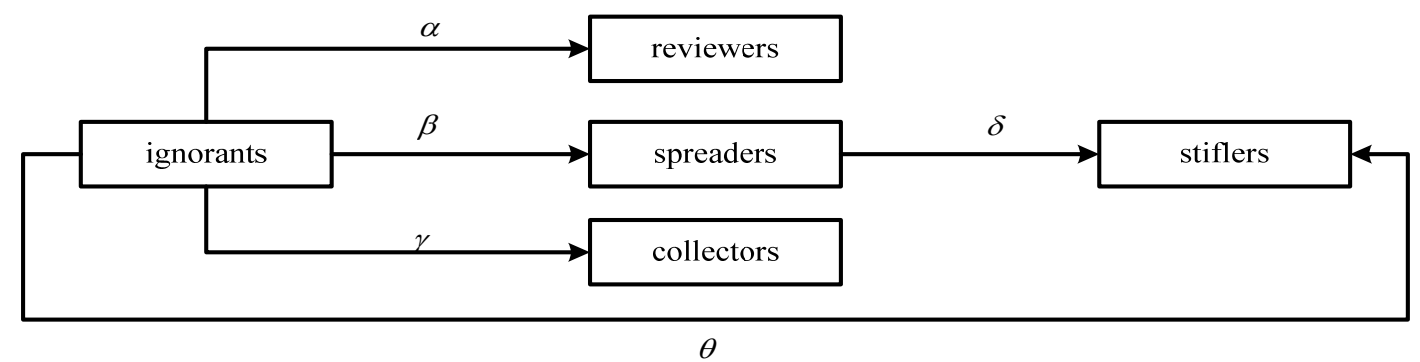

Fig. 1 Structure of the IRC2S information spreading model

As shown in Fig1, the information spreading rules of the IRC2S model can be summarized as follows. (1) When an ignorant encounters a spreader, there are four possible outcomes: (i) the ignorant may comment on the message or give the message thumbs up with probability $\alpha$; (ii) the ignorant may be so interested in the message that he/she shares it in his/her circle of friends with probability $\beta$; (iii) the ignorant deems that the message has collecting value, so he/she collects it

with probability $\gamma$; (iv) In addition, the ignorant may have no response to the message with probability $\theta$. (2) When a spreader encounters another spreader, he/she could think the message has been propagated universally. So the sharer becomes a stifler with probability $\delta$.

Denote density of five groups at time $t$ by $I(t), R(t), S_{1}(t), C(t), S_{2}(t)$ apart. According to above rules, we can use the mean-field equations to describe the IRC $2 S$ information spreading model.

$$
\begin{aligned}
& \frac{d I(t)}{d t}=-(\alpha+\beta+\lambda+\gamma+\theta)\langle k\rangle I(t) S_{1}(t) \\
& \frac{d R(t)}{d t}=\alpha\langle k\rangle I(t) S_{1}(t) \\
& \frac{d S_{1}(t)}{d t}=\beta\langle k\rangle I(t) S_{1}(t)-\delta\langle k\rangle S_{1}(t)^{2} \\
& \frac{d C(t)}{d t}=\gamma\langle k\rangle I(t) S_{1}(t) \\
& \frac{d S_{2}(t)}{d t}=\theta\langle k\rangle I(t) S_{1}(t)+\delta\langle k\rangle S_{1}(t)^{2}
\end{aligned}
$$




\section{The $I R C 3 S$ information spreading model}

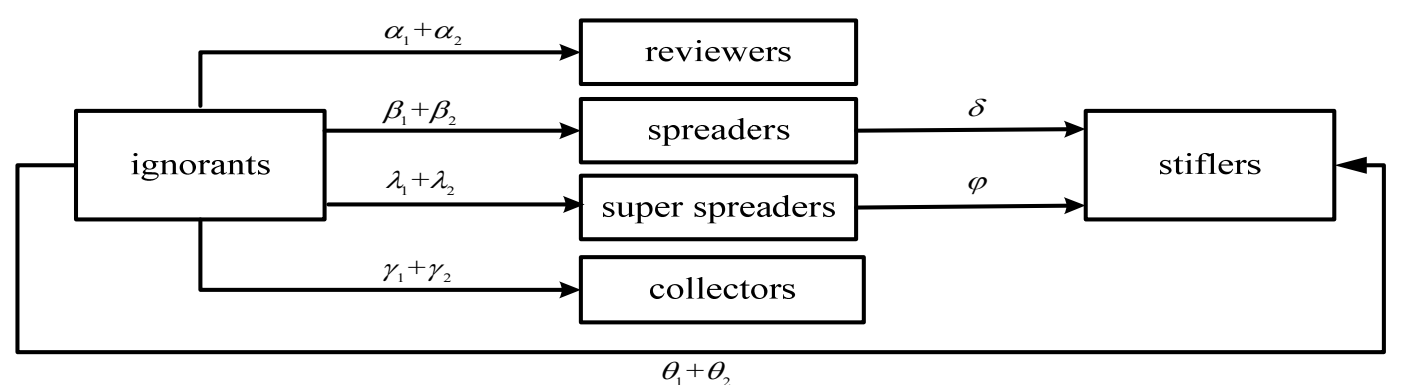

Fig. 2 Structure of the IRC3S information spreading model

Based on the above information spreading model, we can construct a Microblog information spreading model by adding a new group called super spreaders ( $S s$ ), which is called IRC3S

information spreading model. When an ignorant encounter a super spreader, the ignorant will make response with greater probability. Because his/her influence make an ignorant prefer to make response to the information. So the information will spread according to the figure 2 and the following mean-field equations.

$$
\begin{aligned}
\frac{d I(t)}{d t}= & -\left(\alpha_{1}+\beta_{1}+\lambda_{1}+\gamma_{1}+\theta_{1}\right)\langle k\rangle I(t) S_{1}(t)-\left(\alpha_{2}+\beta_{2}+\lambda_{2}+\gamma_{2}+\theta_{2}\right)\langle k\rangle I(t) S s(t) \\
\frac{d R(t)}{d t}= & \alpha_{1}\langle k\rangle I(t) S_{1}(t)+\alpha_{2}\langle k\rangle I(t) S s(t) \\
\frac{d S_{1}(t)}{d t}= & \beta_{1}\langle k\rangle I(t) S_{1}(t)+\beta_{2}\langle k\rangle I(t) S s(t)-\delta\langle k\rangle S_{1}(t)\left(S_{1}(t)+S s(t)\right) \\
\frac{d S s(t)}{d t}= & \lambda_{1}\langle k\rangle I(t) S_{1}(t)+\lambda_{2}\langle k\rangle I(t) S s(t)-\varphi\langle k\rangle S s(t)\left(S_{1}(t)+S s(t)\right) \\
\frac{d C(t)}{d t}= & \gamma_{1}\langle k\rangle I(t) S_{1}(t)+\gamma_{2}\langle k\rangle I(t) S s(t) \\
\frac{d S_{2}(t)}{d t}= & \theta_{1}\langle k\rangle I(t) S_{1}(t)+\theta_{2}\langle k\rangle I(t) S s(t)+\delta\langle k\rangle S_{1}(t)\left(S_{1}(t)+S s(t)\right) \\
& +\varphi\langle k\rangle S s(t)\left(S_{1}(t)+S s(t)\right)
\end{aligned}
$$

\section{The advanced $I R C 3 S$ information spreading model}

The effect of super spreaders not only reflect on the influence but also on the degree. So we classify nodes as center node and ordinary node. It is well known that the degree of center node is bigger than that of ordinary node. We denote the average degree of ordinary nodes and center nodes by $\langle k 1\rangle$ and $\langle k 2\rangle$ separately. So the mean-field equations are modified based on the IRC3S information spreading model as follows.

$$
\begin{aligned}
& \frac{d I(t)}{d t}=-\left(\alpha_{1}+\beta_{1}+\lambda_{1}+\gamma_{1}+\theta_{1}\right)\langle k 1\rangle I(t) S_{1}(t)-\left(\alpha_{2}+\beta_{2}+\lambda_{2}+\gamma_{2}+\theta_{2}\right)\langle k 2\rangle I(t) S s(t) \\
& \frac{d R(t)}{d t}=\alpha_{1}\langle k 1\rangle I(t) S_{1}(t)+\alpha_{2}\langle k 2\rangle I(t) S s(t) \\
& \left.\frac{d S_{1}(t)}{d t}=\beta_{1}\langle k 1\rangle I(t) S_{1}(t)+\beta_{2}\langle k 2\rangle I(t) S s(t)-\delta\langle k 1\rangle S_{1}(t) S_{1}(t)-\delta\langle k 2\rangle S_{1}(t) S s(t)\right)
\end{aligned}
$$




$$
\begin{aligned}
\frac{d S s(t)}{d t} & =\lambda_{1}\langle k 1\rangle I(t) S_{1}(t)+\lambda_{2}\langle k 2\rangle I(t) S s(t)-\varphi\langle k 2\rangle S s(t)\left(S_{1}(t)+S s(t)\right) \\
\frac{d C(t)}{d t}= & \gamma_{1}\langle k 1\rangle I(t) S_{1}(t)+\gamma_{2}\langle k 2\rangle I(t) S s(t) \\
\frac{d S_{2}(t)}{d t}= & \left.\theta_{1}\langle k 1\rangle I(t) S_{1}(t)+\theta_{2}\langle k 2\rangle I(t) S s(t)+\delta\langle k 1\rangle S_{1}(t) S_{1}(t)+\delta\langle k 2\rangle S_{1}(t) S s(t)\right) \\
& +\varphi\langle k 2\rangle S s(t)\left(S_{1}(t)+S s(t)\right)
\end{aligned}
$$

\section{Analysis of numerical simulation}

In this section, Runge-Kutta method is used to solve the mean-field equations. Numerical simulation is performed in a generated homogeneous network which has $10^{5}$ individuals.

Firstly, we perform numerical simulation on the IRC2S information spreading model. We assume average degree of the generated homogeneous network is 10 and $\operatorname{set} \alpha=0.3, \beta=0.3$,

$\lambda=0.01, \gamma=0.09, \theta=0.3, \delta=0.3, \varphi=0.3$. Secondly, numerical simulation is performed on the IRC3S information spreading model. Average degree of the generated homogeneous network is still set to 10 , but set $\alpha_{1}=0.1, \quad \alpha_{2}=0.2, \quad \beta_{1}=0.1, \quad \beta_{2}=0.2, \quad \lambda_{1}=0.003, \quad \lambda_{2}=0.007, \quad \gamma_{1}=0.03, \quad \gamma_{2}=0.06$

\section{$\theta_{1}=0.2, \theta_{2}=0.1, \delta_{1}=0.3, \varphi_{1}=0.3$. Thirdly, we perform numerical simulation on the advanced}

IRC3S information spreading model. We set $\langle k 1\rangle=10,\langle k 2\rangle=1000$ and other parameters are the same with that in IRC3S information spreading model. Density of each group over time of above three models is in Fig. 3 as follows.
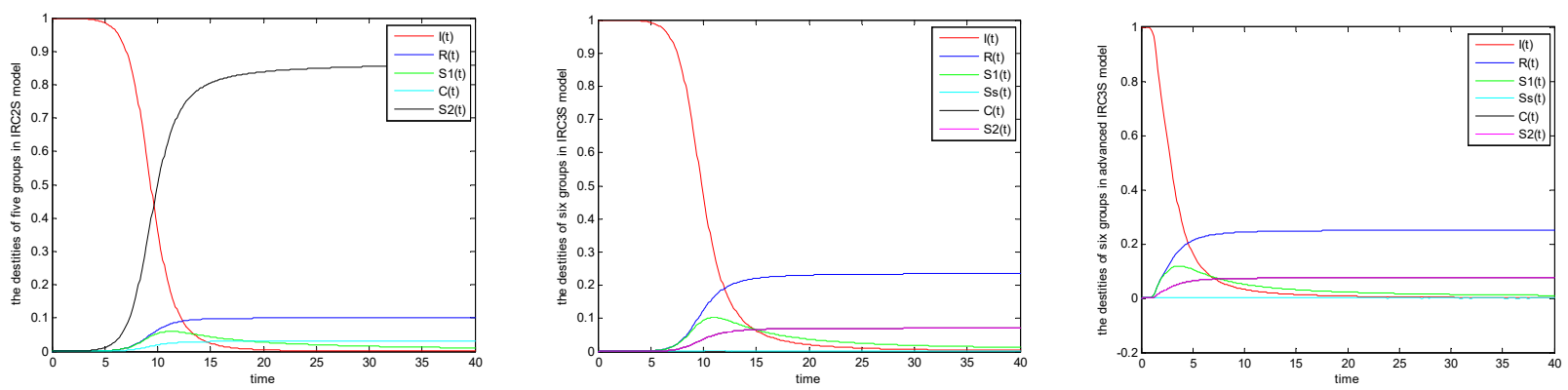

Fig. 3 Density of each group over time in the IRC2S model, in the IRC3S model and in the advanced IRC $3 S$ model respectively

From Fig. 1 and Fig. 2 we can see that the trend of collectors' density over time is similar to that of reviewers'. So we analyze spreading speed and spreading scope only by analyzing density of reviewers and spreaders over time. From Fig. 4 we can see that the peak values of reviewers' and spreaders' density in the IRC $2 S$ model get larger than that in the IRC $3 S$ model. It indicates that influence of super spreader make information spread more widely. 

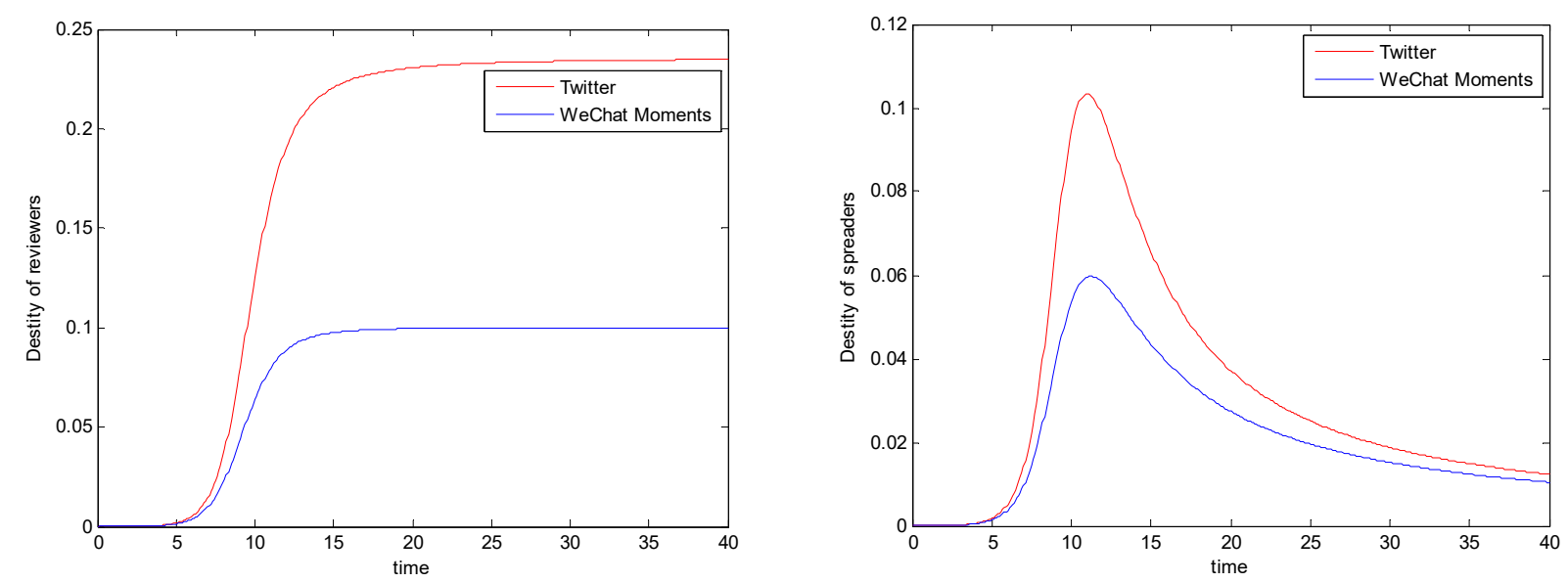

Fig. 4 Compare the densities of reviewers and spreaders over time in the IRC2S model with that in the IRC $3 S$ model

The value of $\langle k 1\rangle$ remain the same, which is 10 . We analyze the effect of average degree on spreading speed and scope by changing the value of $\langle k 2\rangle$. We detect that the time that two groups' density get peak values get shorter when the value of $\langle k 2\rangle$ get larger from Fig. 5. In other words, the larger of the values of $\langle k 2\rangle$, the faster of spreading speed. In addition, the peak values also get large when the average degree of super spreader get large, but the influence is very little.

\section{Summary}

On one hand, influence of super spreaders make ignorant who encounter the information spread by super spreaders more interested in the information so that he/her spread, reviewer or collect the information. So it make information spread widely. On the other hand, because degree of center nodes is bigger than ordinary nodes', spreading speed of information get faster. Overall, centralization of network makes information spread faster and wider as Fig. 6 implies. This is the reason that many news come out in microblog first but not in WeChat Moments.
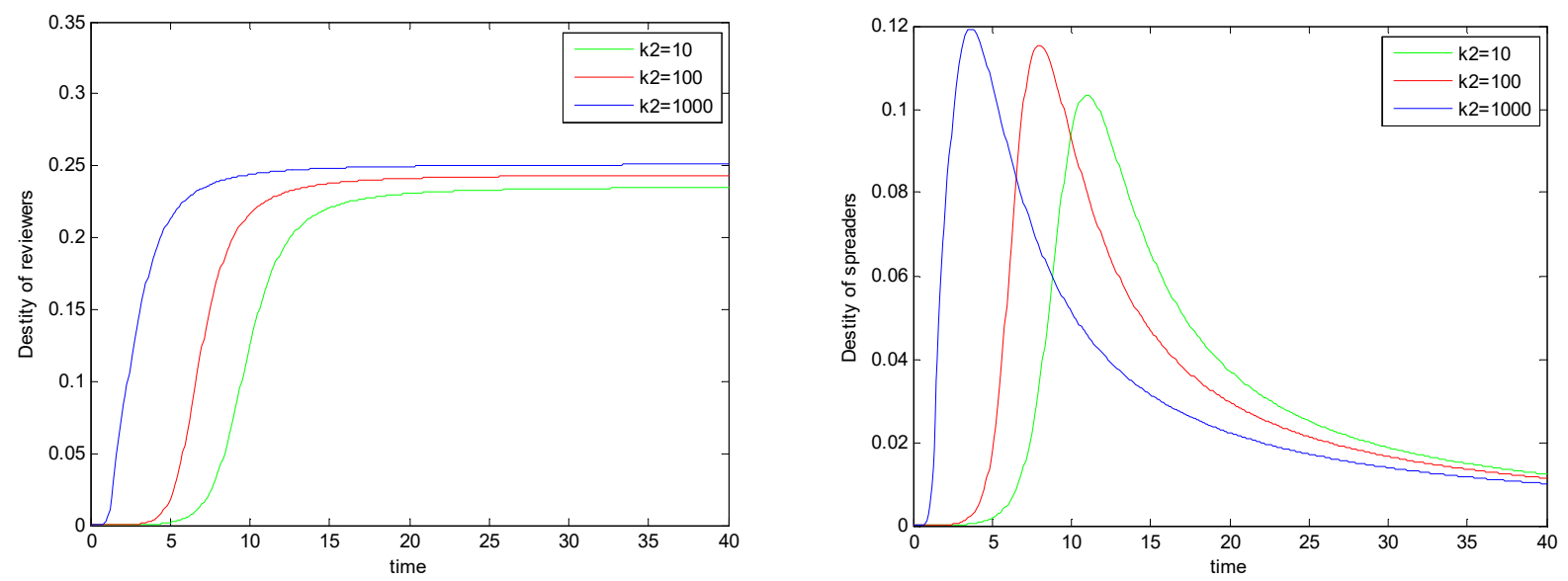

Fig. 5 Densities of reviewers and spreaders over time under different $\langle k 2\rangle$ in the IRC $3 S$ model 

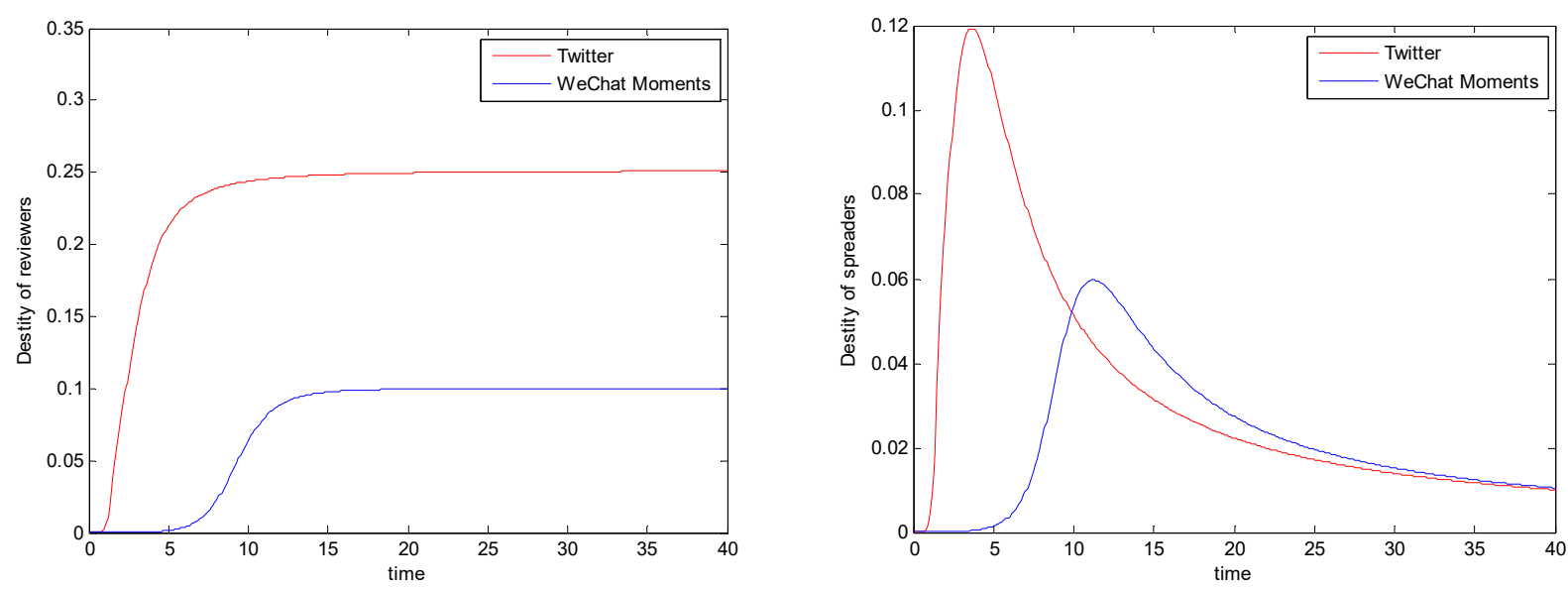

Fig. 6 Compare the densities of reviewers and spreaders over time in the IRC2S model with that in the advanced IRC $3 S$ model.

\section{References}

[1] D.H. Zanette, Dynamics of rumor propagation on small-world networks, Physical Review E 65 (2002).

[2] L. Zhao, X. Qiu, X. Wang, Rumor spreading model considering forgetting and remembering mechanisms in homogeneous networks, PhysicaA 392 (2012) 987-994.

[3] Yaqi Wang, Xiaoyuan Yang, Yiliang Han, Xuan Wang, Rumor spreading model with trust mechanism in complex social networks, Commun. Theor. Phys.59 (2013) 510-516.

[4] J Zhao, J Wu, X Feng, H Xiong, $\mathrm{K} \mathrm{Xu}$, Information propagation in online social networks: a tie-strength perspective, Knowledge \& Information Systems 32 (2012) 589-608.

[5] Yaming Zhang, Chaosheng Tang, Weigang Li, Cooperative and competitive dynamics model for information propagation in online social networks, Journal of Applied Mathematics 2014 (2014) 610382.

[6] LaiJun Zhao, Hongxin Cui, SIR rumor spreading model in the new media age, Physica A 392 (2013) 995-1003. 